\title{
Detection of cardiac sounds components: a pilot study
}

\author{
Norezmi Jamal $^{1}$, Nabilah Ibrahim ${ }^{2}$, MNAH Sha'abani ${ }^{3}$, Zulkifli Taat ${ }^{4}$ \\ ${ }^{1,2}$ Faculty of Electrical and Electronic Engineering, Universiti Tun Hussein Onn Malaysia, Malaysia \\ ${ }^{3}$ Centre of Diploma Studies, Universiti Tun Hussein Onn Malaysia, Malaysia \\ ${ }^{4}$ Pusat Kesihatan Universiti, Universiti Tun Hussein Onn Malaysia, Malaysia
}

\begin{tabular}{|c|c|}
\hline Article Info & ABSTRACT \\
\hline & This paper presents a preliminary study related to the detection and \\
\hline Received Jun 18, 2019 & $\begin{array}{l}\text { identification of cardiac sounds components including first sound (S1), } \\
\text { second sound (S2) and murmurs. Detection and identification of cardiac }\end{array}$ \\
\hline Revised Sep 19, 2019 & sounds are an important process in automated cardiac sound analysis system \\
\hline Accepted Oct 3, 2019 & $\begin{array}{l}\text { in order to automatically diagnose people who are having cardiovascular } \\
\text { disorder and determine the existence of murmurs. Sixteen of recorded cardiac }\end{array}$ \\
\hline Keywords: & $\begin{array}{l}\text { sounds (eight normal cardiac sounds, four abnormal cardiac sounds with } \\
\text { systole murmur, and four abnormal cardiac sounds with diastole murmur) }\end{array}$ \\
\hline Cardiac sounds & from PASCAL Classifying Heart Sounds Challenge database were examined \\
\hline Detection & $\begin{array}{l}\text { for analysis. This work is significant in studying the time and time-frequency } \\
\text { based detection of cardiac sounds components characteristics. In time-based }\end{array}$ \\
\hline Envelope-based approach & analysis, envelope of signal energy was used to do the peak detection of $\mathrm{S} 1$, \\
\hline $\begin{array}{l}\text { Fourier synchrosqueezed } \\
\text { transform }\end{array}$ & $\begin{array}{l}\mathrm{S} 2 \text { and murmur and also analysis of cardiac cycle, systole and diastole } \\
\text { duration. While time-frequency based analysis was used to determine the } \mathrm{S} 1 \text {, }\end{array}$ \\
\hline Phonocardiogram & $\begin{array}{l}\text { S2 and murmur frequency range. The findings yield the overall accuracy of } \\
\text { envelope-based detection for normal cardiac sound signal at } 60.85 \% \text { while } \\
\text { for abnormal cardiac sound signal at } 57.24 \% \text {. }\end{array}$ \\
\hline
\end{tabular}

Copyright $\odot 2020$ Institute of Advanced Engineering and Science. All rights reserved.

\section{Corresponding Author:}

Nabilah Ibrahim,

Faculty of Electrical and Electronic Engineering,

Universiti Tun Hussein Onn Malaysia,

86400 Parit Raja, Johor, Malaysia.

Email: nabilah@uthm.edu.my

\section{INTRODUCTION}

Cardiac murmurs are abnormal cardiac sound during heartbeat cycle. They may differ in terms of timing, location, intensity, quality and positional changes [1-2]. They are produced from valve defects that can conventionally be heard with an acoustic stethoscope. They are rasping sound due to the generation of vibration caused by the abnormal movement of blood within or between cardiac chambers, or by turbulent flow within the pulmonary artery or aorta just distal to the outflow valve [2]. Towards modern technology, cardiac sounds including first sound (S1), second sound (S2) and murmurs can be heard and visualized via digital stethoscope which is known as phonocardiogram (PCG). To be noted here, S1 is defined as "Lub" caused by the tricuspid and mitral (bicuspid) valve, while S2 is defined as "Dub" caused by the aortic and pulmonary valve [2]. If a murmur exists in systole which is in cardiac ventricle contraction mode, between the S1 and S2 cardiac sounds, it is known as a "systolic murmur." If it exists in diastole, which is in cardiac ventricle relaxation mode (between S2 and S1), it is known as a "diastolic murmur" [2].

In recent years, the detection of S1, S2 and murmurs can be realized automatically through signal processing approach and mathematical algorithm [3-5]. It is an essential process in pre-processing stage before proceeding to the feature extraction and classification stage for automated cardiac sound analysis system as shown in Figure 1 [3]. Initially, a physician captures and records cardiac sounds using an electronic stethoscope which is embedded with the amplifier and filter circuit. Then, the cardiac sound signal is 
converted into digital signals that can be read by the computer. Next, the cardiac sound signal is sampled, segmented, filtered and analyzed with the sampling frequency in signal pre-processing process. The last process of automated cardiac sound analysis system is signal post-processing and analysis which consists of feature extraction and classification techniques.

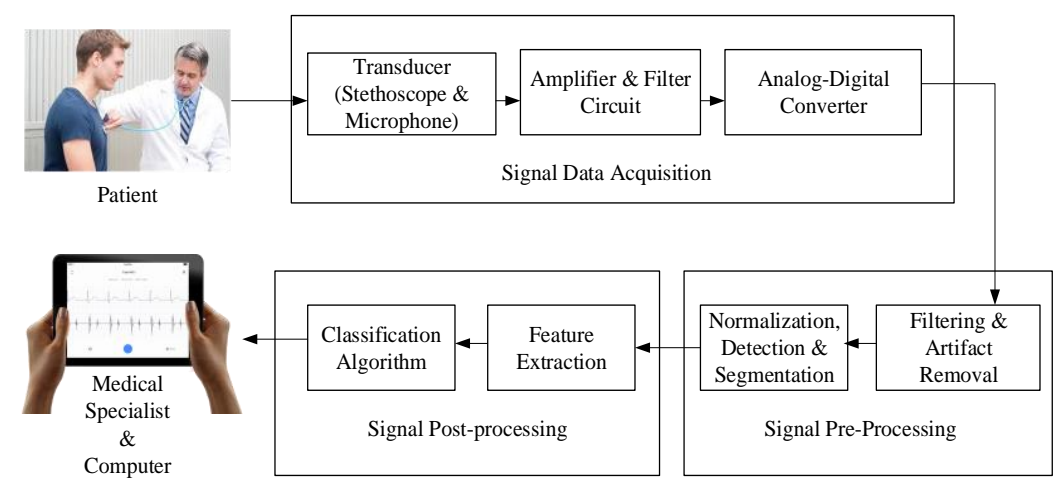

Figure 1. A general block diagram of automated cardiac sound analysis system [3]

A considerable amount of literature has already been published on envelope-based detection of S1, S2 and murmur, which used envelope of Shannon energy to determine the distinct peaks either S1 or S2 in time series [6-12]. Most of them used Shannon energy in envelope-based approach due to its advantages can increase the medium intensity signal and attenuates the result of low intensity signal to the high intensity signal [13]. However, it suffers a lot of faulty detections when applying the threshold to detect the peak signal [14]. Authors in [6] claimed that S1 and S2 were detected when using low pass finite impulse response (FIR) filter with $100 \mathrm{~Hz}$ of cut-off frequency, but it will cause the elimination of murmur. Then, [7-8] articles are only limited to analysis of S1 and S2 duration without analysis of systole and diastole duration. On the other hand, authors in [7, 9, 10-11] focused on the detection of S1 and S2 of cardiac sound signal without murmur detection. Otherwise, authors in [9-10, 12] used wavelet transform for denoising of cardiac sound signal in S1 and S2 detection. The difficulty of that approach is to adjust a few thresholds for reconstruction of the cardiac sound signal [14]. Moreover, other study used external reference such as Electrocardiogram (ECG) signal for detection of S1 and S2 in PCG signal [15].

Due to the aforementioned constraints, the aim of this paper is to study cardiac sound signal components that include S1, S2 and murmur characteristics detection in time and time-frequency (T-F) domain analysis. A squared energy envelope is proposed in this paper because of its better performance compared to Shannon energy envelope-based approach with the peak conditioning for time domain analysis [16]. While Fourier synchro squeezed transform is used for time-frequency domain analysis. So, the paper is organized as follows: Section 2 discusses the methods or steps of research work. Next, Section 3 presents the results and briefly discusses the findings and finally Section 4 is devoted to conclusions.

\section{RESEARCH METHOD}

In this study, eight audios of normal and abnormal cardiac sounds from PASCAL Classifying Heart Sounds Challenge [17] were used for analysis in time and time-frequency (T-F) domain, respectively. The abnormal cardiac sounds consist of four cardiac sounds with systole murmur and other four cardiac sounds with diastole murmur. The steps of research work for time domain analysis are illustrated in Figure 2 by firstly reading the audio file to get raw data and frequency sampling, fs. Since the frequency sampling of cardiac sound signal is $44100 \mathrm{~Hz}$ which leads to the computational burden, so, the signal was down-sampled into $2000 \mathrm{~Hz}$ [18]. Next, bandpass Butterworth filter with five order and cut off frequency between 25 and $250 \mathrm{~Hz}$ were chosen due to frequency range of S1 and S2 events [3]. Then, the signal, x(i) was normalized to ensure the peak signal is more prominent as shown in (1);

$$
x_{\text {norm }}(i)=\frac{x(i)}{\max (|x(i)|)}
$$


where $x(i)$ is the original signal and xnorm (i) is normalized signal. After that, signal energy, s(i) of normalized signal was calculated as shown in (2). This is known as squared energy, which is chosen in this research work since the intensity of S1 and S2 are significantly different to be identified with linear threshold for peak conditioning [16].

$$
s(i)=\sum_{i=1}^{N}\left(x_{\text {norm }}(i)\right)^{2}
$$

Signal energy was enveloped to find energy peak of S1, S2 and murmur. All the stationary noise signal energy, which is less than 0.03 was removed in smoothing process since S1 and S2 energy peak are basically greater than that value [19]. The reason is to reduce false detection of cardiac sound signal. In this paper, upper and lower threshold were pre-defined, where the upper threshold, up was firstly set to $70 \%$ of maximum peak while lower threshold, lo, was set to $0 \%$ of maximum peak for normal cardiac sound signal case. But, for abnormal cardiac sound signal case, the lower threshold was pre-defined as $30 \%$ of maximum peak. Then, these thresholds were manually adjusted based on the current peak energy signal of S1, S2 and murmur for different recorded cardiac sound file as illustrated in Table 1. If the energy peak signal is greater than upper threshold, S1 was detected, while if energy peak signal is greater than lower threshold and lower than upper threshold, then, S2 was detected. Otherwise, murmur was detected if the peak energy is lower than lower threshold.

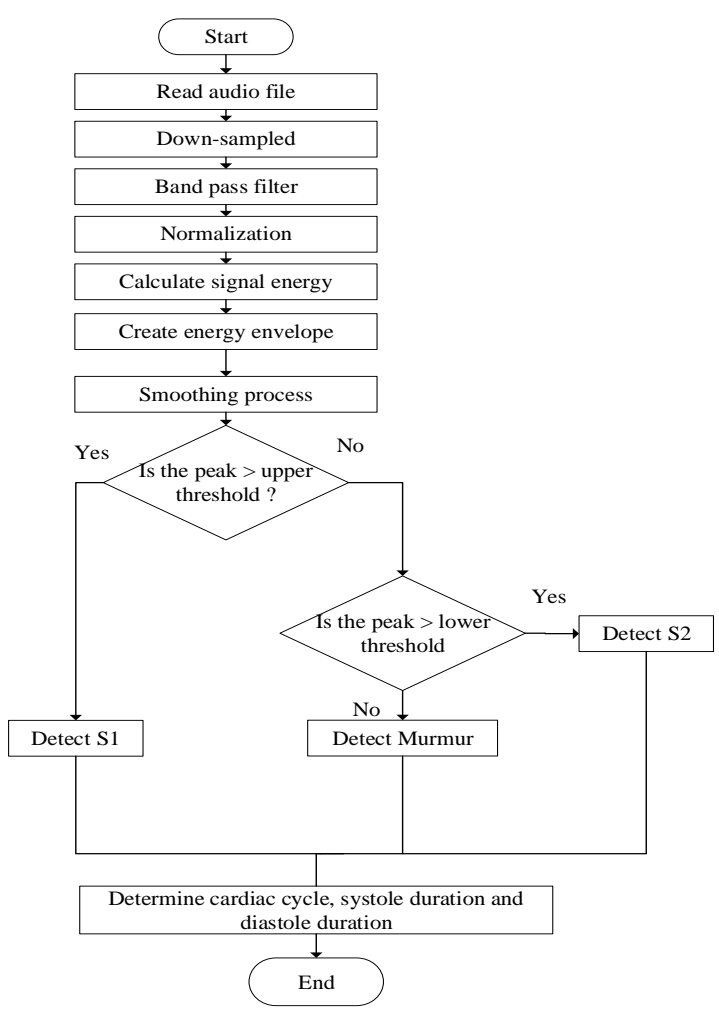

Figure 2. Flowchart of S1, S2 and murmur cardiac sounds detection using energy envelope-based detection

For T-F domain analysis, the filtered and normalized cardiac sound signal was analyzed using Fourier synchro squeezed transform, see Figure 3. The advantage of this approach is the time-frequency of signal event information is known and time-scale representation is sharpened. This was realized using fsst function in Matlab. The time series of cardiac sound signal was transformed into short-time Fourier transform with hanning window. The transform values were then "squeezed" so that they concentrate around curves of instantaneous frequency in the time-frequency plane [20]. The resulting synchrosqueezed transform was shown in (3). 


$$
T_{g} f(t, \omega)=\int_{-\infty}^{\infty} V_{g} f(t, \eta) \delta\left(\omega-\Omega_{g} f(t, \eta)\right) d \eta
$$

where is short-time Fourier transform of a function, f, using a spectral hanning window, g with $0.13 \mathrm{~s}$ length and is instantaneous frequencies that are estimated with the phase transform [20].

Table 1. Threshold Variation based on Different Cardiac Sound Types

up $=70$
To=0

up $=70$

$10=30$

$10=10$ (b) Systole murmur

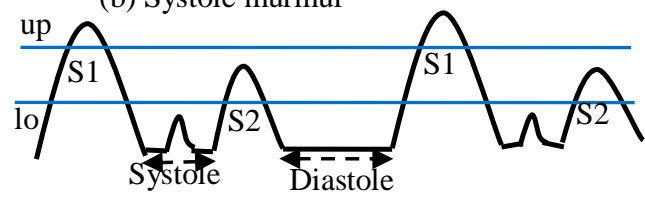

(c) Diastole murmur

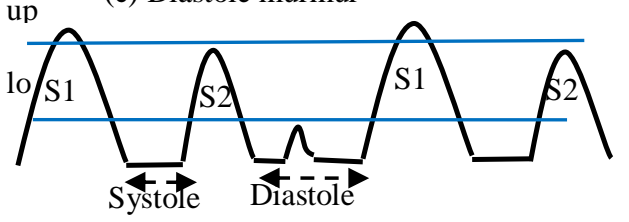

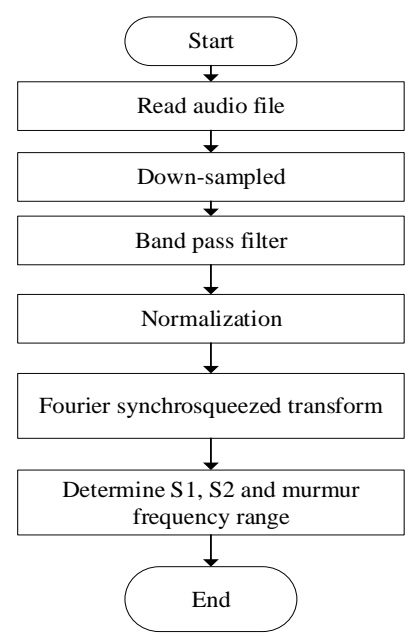

Figure 3. Flowchart of S1, S2 and murmur cardiac sounds detection using Fourier synchrosqueezed transform

\section{RESULTS AND ANALYSIS}

Figure 4 (a) shows the peak detection of S1 and S2 in normal cardiac sound signal based on the envelope of normalized signal energy for one of cardiac sound data from PASCAL database. While the peak detection of abnormal cardiac sound with systole and diastole murmur in time domain are shown in Figure 4(b) and Figure 4 (c), respectively. From Figure 4(a), Figure 4(b) and Figure 4(c), the red circle indicates S1 peak energy level if the peak is greater than upper threshold while the black circle refers to S2 
peak energy level, if it is lower than upper threshold. Then, the blue circle indicates murmur peak energy level if the peak lower is than lower threshold. Based on time domain analysis, the murmur detection is a challenging task to do using peak conditioning approach when its energy level is approximately similar to the noise energy level. On the other hand, murmur energy peak might be vanished during smoothing process that will cause incorrect detection. Since there is approximately similar energy peak between noise and murmur energy, it is difficult to be determined in time series. For validation of murmur detection, it is analyzed using Fourier synchro squeezed transform as shown in Figure 4(d)-(f). For normal cardiac sound signal case, it is illustrated in Figure 4(d). As for abnormal cardiac sound signal case, it is illustrated in Figure 4(e) and Figure 4(f), which are cardiac sound signal with systole and diastole murmur, respectively.
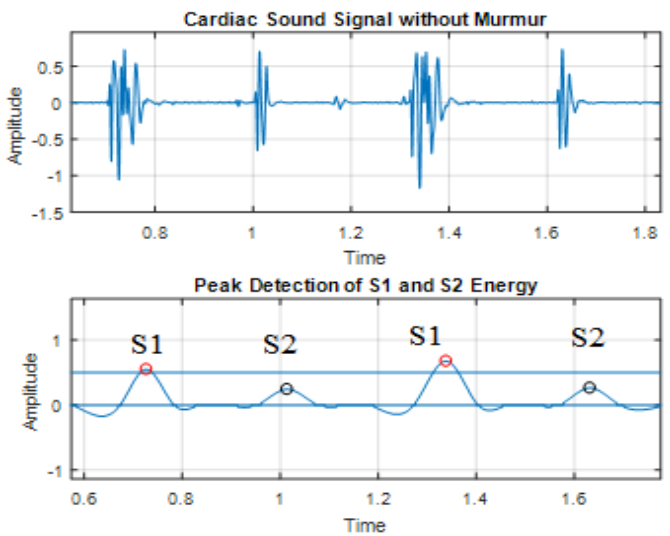

(a)
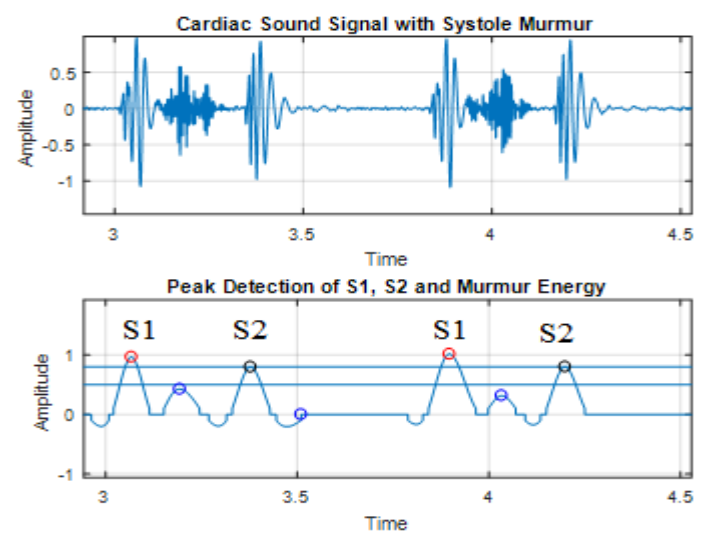

(b)
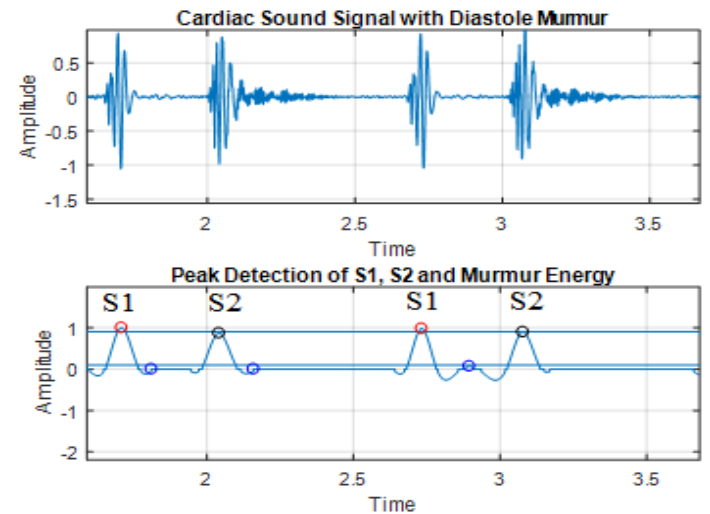

(c)

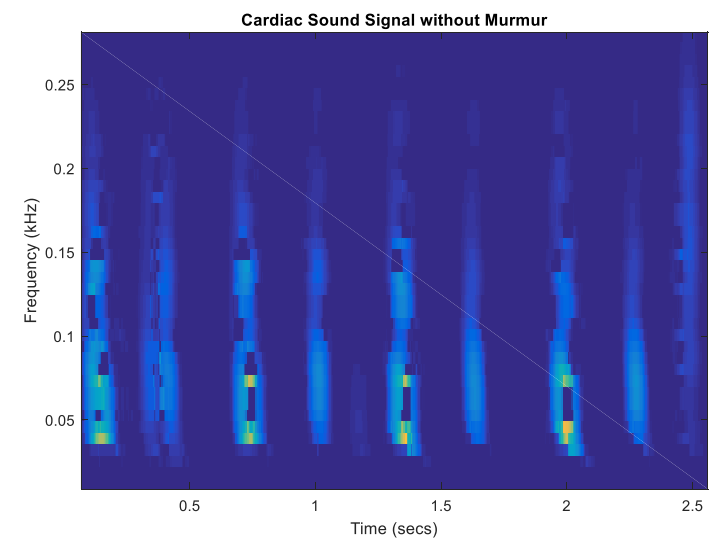

(d)

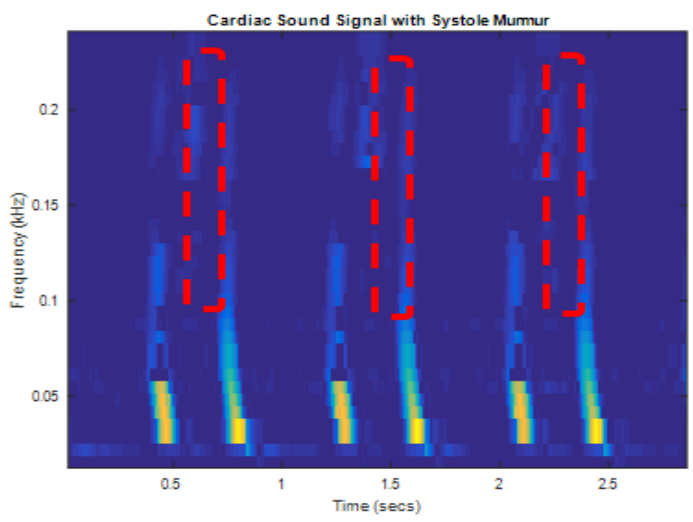

(e)

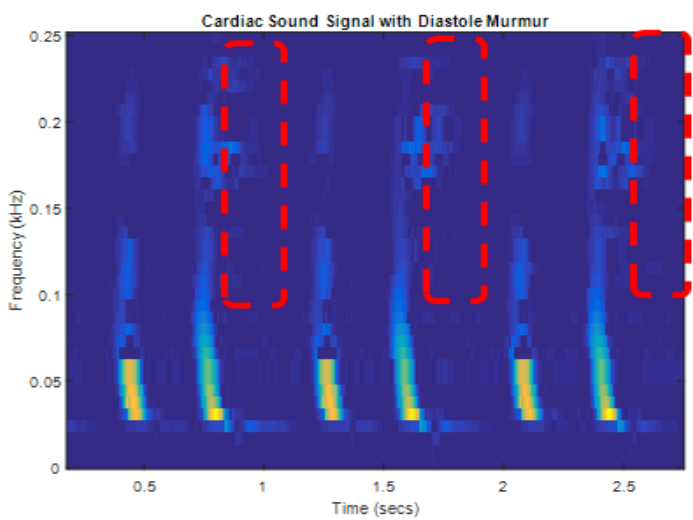

(f)

Figure 4. S1, S2 and murmur cardiac sounds detection using envelope of signal energy and Fourier synchro squeezed transform 
It can be seen that S1, S2 and murmurs are clearly identified based on T-F domain. There are time and frequency information for S1, S2 and murmur events. The murmur events are identified by red dashed line. The different color includes dark blue, light blue and yellow denotes spectral power. The yellow color denotes the higher power, and the dark blue color denotes the lower power, and it can be seen clearly that the frequency content changes during the heartbeat cycle. S1 of cardiac sound signal produces higher spectral power compared to S2 for normal and abnormal cardiac sound signal.

Table 2(a) shows the summary of duration of cardiac cycle, systole, and diastole in time domain analysis. S1 and S2 frequency range are also identified in T-F domain analysis for eight data of normal cardiac sound signal. While Table 2(b) shows the duration summary of cardiac cycle, systole, and diastole in time domain analysis for abnormal cardiac sound. It also tabulates S1, S2 and murmur frequency range in TF domain analysis for eight data of abnormal cardiac sound signal. The average of cardiac cycle value for normal cardiac sound signal is $0.73 \mathrm{~s}$ while the average value for abnormal cardiac cycle is $0.87 \mathrm{~s}$, which is much longer. This finding agreed with other study that claimed the cardiac cycle varied with the presence of cardiac murmurs [6]. Then, the average duration of diastole is longer than the duration of the systole for normal and abnormal cardiac sound signal, which are $0.33 \mathrm{~s}$ and $0.39 \mathrm{~s}$ respectively, which is also supported by [21]. To be noted here, the duration of diastole for abnormal cardiac sound signal is longer compared to normal cardiac sound signal with 0.06 s of time difference due to the existence of murmurs.

Since time domain analysis can only obtain the time information of cardiac sound signal event, the analysis of frequency range for S1, S2 and murmur had been obtained using Fourier synchro squeezed transform as mentioned in Table 2(a) and (b) for normal and abnormal cardiac sound signal respectively from manual observation. The average of frequency range of $\mathrm{S} 1$ for normal cardiac sound signal is between 29 and $230 \mathrm{~Hz}$ while for abnormal cardiac sound signal is between 27 and $229 \mathrm{~Hz}$. Next, S2 frequency range is between 35 and $195 \mathrm{~Hz}$ for normal cardiac sound signal while from 25 to $226 \mathrm{~Hz}$ is produced by abnormal cardiac sound signal. To be noted here, there are overlapping frequency among S1, S2 and murmur frequency, which the murmur frequency range is located between frequency range of S1 and S2 cardiac sound signal, with the average value is between 75 and $235 \mathrm{~Hz}$ for abnormal cardiac sound signal. This finding agreed with the theoretical study on the cardiac sound and murmur frequency range, which are between $24 \mathrm{~Hz}$ and $1024 \mathrm{~Hz}$ as stated in [3, 22].

The evaluation of energy envelope-based detection performance was presented using confusion matrix as shown in Table 3 and Table 4 for normal and abnormal cardiac sound signal respectively. The classification of S1, S2 and murmur class is based on input of maximum peak energy for each class using human observation on the labelled energy peak. From the confusion matrix tables, the diagonal elements of the matrix have been highlighted with blue color for both cases. The diagonal cells contain the number of correctly identified S1, S2 and murmurs maximum peaks. So, the performance of energy envelope-based detection was measured based on the overall accuracy, OA, which is the ratio of total of diagonal cells to the number of samples, $n$ as shown in (4).

$$
O A=\frac{\sum_{k=1}^{k=K} \text { cell }_{k}}{n}
$$

As mentioned in Table 3, the number of samples for normal cardiac sounds are 235, which are counted by the total number of S1 and S2 from eight normal recorded sounds. The number of correctly detected S1 is 69 counts while the number of correctly detected S2 is 74 counts. The fault detection of S1 is high because its energy peak is approximately similar to that of S2. Moreover, it is also contributed by S1 split sound, that occurred by the adjournment of the tricuspid valve closure [23] or wider S2 split [24]. Conclusively, it is difficult to correctly detect either S1 or S2 peak using peak conditioning approach. The result exhibits the overall accuracy for detection of S1 and S2 of cardiac sound signal is $60.85 \%$ for normal case. For abnormal cardiac sound signal detection case as shown in Table 4, the overall accuracy produced is $57.24 \%$, where $\mathrm{S} 1$ is 64 counts, S2 is 56 counts and murmurs are 50 counts with 297 of samples. To be noted here, there is incorrect detection of murmur class when the energy peak signal is lower than lower threshold since the total number of samples is different with the prediction value. So, the result of abnormal cardiac sound detection is lower compared to that of normal cardiac sound. The reason is the detection process based on peak conditioning approach become a challenging task when dealing with the murmur. There is false detection for murmur detection especially in case of systole murmur as well as diastole murmur. This is because of murmur energy level is approximately similar to the noise energy level. In short, envelope-based detection approach suffered from misdetection issue when there are noises and peak variations [25]. 
Table 2. Components of Normal and Abnormal Cardiac Sound Signal Characteristics in Time and TimeFrequency Domain Analysis

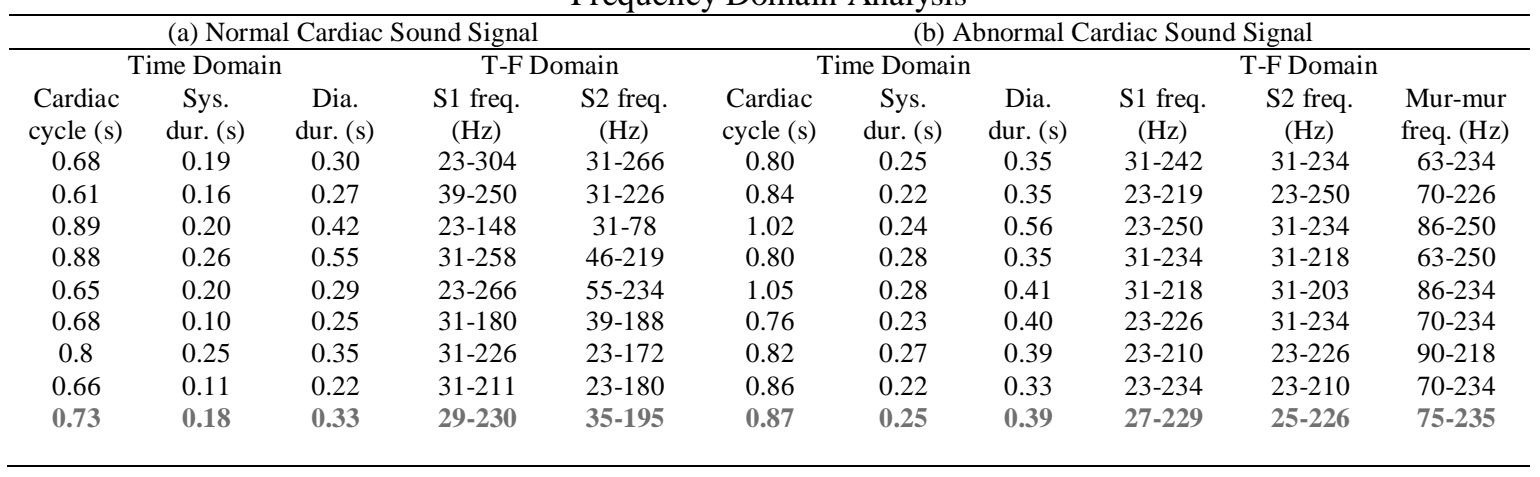

Table 3. Confusion matrix for normal cardiac

\begin{tabular}{|c|c|c|c|c|}
\hline \multicolumn{5}{|c|}{ sound detection } \\
\hline \multirow{4}{*}{ 异 } & $n=235$ & S1 & S2 & Sum \\
\hline & $\mathrm{S} 1$ & 69 & 11 & 80 \\
\hline & S2 & 81 & 74 & 155 \\
\hline & $\begin{array}{l}\text { Sum } \\
\text { Overall Accuracy }\end{array}$ & 150 & $\begin{array}{c}85 \\
60.85 \%\end{array}$ & \\
\hline
\end{tabular}

Table 4. Confusion matrix for abnormal cardiac

\begin{tabular}{|c|c|c|c|c|c|}
\hline \multicolumn{6}{|c|}{ sound detection } \\
\hline & & \multirow{2}{*}{\multicolumn{3}{|c|}{ S2 Murmur }} & \\
\hline \multirow{6}{*}{ एٕ } & $n=297$ & & & & \multirow{5}{*}{$\begin{array}{c}\text { Sum } \\
71 \\
70 \\
51\end{array}$} \\
\hline & S1 & 64 & 7 & 0 & \\
\hline & S2 & 11 & 56 & 3 & \\
\hline & Murmur & 0 & 1 & 50 & \\
\hline & Sum & 75 & 64 & 53 & \\
\hline & Overall Accuracy & & & $4 \%$ & \\
\hline
\end{tabular}

\section{CONCLUSION}

The cardiac sound components characteristics and envelope-based detection approach have been investigated for normal and abnormal cardiac sound signals respectively. For cardiac sound components characteristics, the result shows that the average of cardiac cycle for abnormal cardiac sound was longer compared to normal cardiac sound due to the existence of murmur. Since time-based analysis only have the time information of S1, S2 and murmur event, Fourier synchrosqueezed transform has been used to identify the time-frequency information of S1, S2 and murmur events. While for enveloped-based peak detection of cardiac sounds include S1, S2 and murmur, the normal cardiac sound produced higher overall accuracy compared to the abnormal cardiac sound with $60.85 \%$ and $57.24 \%$, respectively. This value of accuracy is acceptable with the number of samples. As number of samples increase, the accuracy value also will be increased unless there is less distorted data. Moreover, this study is only limited to the cardiac sounds' envelope-based detection based on peak conditioning rule with manual threshold adjustment, thus, it is possible to do the adaptive approach and other methods like feature-based approach to improve the performance of automated cardiac sound analysis.

\section{ACKNOWLEDGEMENTS}

Highly appreciation to Universiti Tun Hussein Onn Malaysia (UTHM) and Ministry of Higher Education for funding this research work under Fundamental Research Grant Scheme (K048) and Postgraduate Research Grant (U712).

\section{REFERENCES}

[1] A.N. Pelech. "The physiology of cardiac auscultation". Pediatric Clinics, 51(6), 2004, p.1515-1535.

[2] R.E. Klabunde. "Cardiovascular physiology concepts". Lippincott Williams and Wilkins. Baltimore, 2011.

[3] A.K. Abbas and R. Bassam. "Phonocardiography signal processing". Synthesis Lectures on Biomedical Engineering, 4(1), 2009, p.1-194.

[4] S. Leng, R. San Tan, K.T.C. Chai, C., Wang, D. Ghista and L. Zhong. "The electronic stethoscope". Biomedical engineering online, 14(1), 2015, p.66.

[5] B.S. Emmanuel. "A review of signal processing techniques for heart sound analysis in clinical diagnosis". Journal of medical engineering and technology, 36(6), 2012, p. 303-307.

[6] A. Atbi and S.M. Debbal. "Segmentation of pathological signals phonocardiogram by using the Shannon energy envelogram". AJCM, 2(1), 2013, p.1-14.

[7] L.C. Hamza., S.M. Debbal and F. Bereksi-Reguig. "Segmentation of heart sounds and heart murmurs". Journal of Mechanics in Medicine and Biology, 8(04), 2008, p.549-559. 
[8] E.F. Gomes and E. Pereira. "Classifying heart sounds using peak location for segmentation and feature construction”. In Workshop Classifying Heart Sounds, La Palma, Canary Islands, 2012.

[9] F. Meziani, S.M. Debbal and A. Atbi. "Analysis of phonocardiogram signals using wavelet transform". Journal of Medical Engineering and Technology, 36(6), 2012, p.283-302.

[10] X. Wang, Y. Li, C. Sun and C. Liu. "Detection of the first and second heart sound using heart sound energy". In 2nd International Conference on Biomedical Engineering and Informatics, p.1-4. IEEE, 2009.

[11] F. Chakir, A. Jilbab, C. Nacir, A. Hammouch and A.H. El Hassani. 2016, May. "Detection and identification algorithm of the S1 and S2 heart sounds". In Electrical and Information Technologies (ICEIT), 2016 International Conference on (pp. 418-420). IEEE.

[12] V.N. Varghees, K.I. Ramachandran, and K.P. Soman. "Wavelet-based fundamental heart sound recognition method using morphological and interval features". Healthcare Technology Letters, 5(3), 2018, p.81-87.

[13] S. Choi, and Z. Jiang. "Comparison of envelope extraction algorithms for cardiac sound signal segmentation". Expert Systems with Applications, 34(2), 2008, p.1056-1069.

[14] N. Marques, R. Almeida, A.P. Rocha, and M. Coimbra. "Exploring the Stationary Wavelet Transform detail coefficients for detection and identification of the S1 and S2 heart sounds". In Computing in Cardiology 2013, pp. 891-894, IEEE, 2013.

[15] F. Safara. "Cumulant-based trapezoidal basis selection for heart sound classification". Medical and biological engineering and computing, 53(11), 2015, pp. 1153-1164.

[16] C. Liu, D. Springer, Q. Li, B. Moody, R.A. Juan, F.J. Chorro, F. Castells, J.M. Roig, I. Silva, A.E. Johnson and Z. Syed. "An open access database for the evaluation of heart sound algorithms". Physiological Measurement, 37(12), 2016, p.2181.

[17] P.J. Bentley, G. Nordehn, M. Coimbra, S. Mannor, R. Getz. "The PASCAL Classifying Heart Sounds Challenge 2011", www.peterjbentley.com/heartchallenge, Accessed November,5,2018.

[18] A. Hamidah, R. Saputra, T.L. Mengko, R. Mengko, and B. Anggoro. "Effective heart sounds detection method based on signal's characteristics". In 2016 International Symposium on Intelligent Signal Processing and Communication Systems (ISPACS) (pp. 1-4). IEEE, 2016.

[19] Q. Liu, X. Wu and X. Ma. "An automatic segmentation method for heart sounds". Biomedical engineering online, 17(1), 2018, p.106.

[20] T. Oberlin, S. Meignen, and V. Perrier. "The Fourier-based synchrosqueezing transform". In IEEE International Conference on Acoustics, Speech and Signal Processing (ICASSP), p. 315-319, IEEE, 2014.

[21] M.V. Shervegar and G.V. Bhat. "Automatic segmentation of phonocardiogram using the occurrence of the cardiac events". Informatics in Medicine Unlocked, 9, 2017, p.6-10.

[22] D. Boutana, M. Benidir, and B. Barkat. "Segmentation and identification of some pathological phonocardiogram signals using time-frequency analysis". IET signal processing, 5(6), 2011, p. 527-537.

[23] S. J. Coviello. "Auscultation Skills: Breath and Heart Sounds". Lippincott Williams and Wilkins, 2013.

[24] V. N. Varghees and K. I. Ramachandran. "Effective heart sound segmentation and murmur classification using empirical wavelet transform and instantaneous phase for electronic stethoscope". IEEE Sensors Journal, 17(12), 2017, p. 3861-3872.

[25] S. I. Malik, M. U. Akram, and I. Siddiqi. "Localization and classification of heartbeats using robust adaptive algorithm". Biomedical Signal Processing and Control, 49, 2019, 57-77. 